\title{
Safety and efficacy of ustekinumab or golimumab in patients with chronic sarcoidosis
}

\author{
Marc A. Judson ${ }^{1}$, Robert P. Baughman², Ulrich Costabel ${ }^{3}$, Marjolein Drent ${ }^{4}$, \\ Kevin F. Gibson ${ }^{5}$, Ganesh Raghu ${ }^{6}$, Hidenobu Shigemitsu7,8, Joseph B. Barney ${ }^{9}$, \\ Daniel A. Culver ${ }^{10}$, Nabeel Y. Hamzeh ${ }^{11}$, Marlies S. Wijsenbeek ${ }^{12}$, Carlo Albera ${ }^{13}$, \\ Isham Huizar ${ }^{14}$, Prasheen Agarwal ${ }^{15}$, Carrie Brodmerkel ${ }^{16}$, Rosemary Watt ${ }^{17}$ \\ and Elliot S. Barnathan ${ }^{17}$
}

\begin{abstract}
Affiliations: 'Dept of Medicine, Albany Medical College, Albany, NY, USA. ${ }^{2}$ Dept of Internal Medicine, University of Cincinnati Medical Center, Cincinnati, $\mathrm{OH}, \mathrm{USA} .{ }^{3}$ Ruhrlandklinik and University of Duisburg-Essen, Essen, Germany. ${ }^{4}$ Dept of Interstitial Lung Diseases, Gelderse Vallei Hospital, Ede, The Netherlands. ${ }^{5}$ Division of Pulmonary, Allergy and Critical Care Medicine, University of Pittsburgh, Pittsburgh, PA, USA. ${ }^{6}$ Division of Pulmonary and Critical Care Medicine, University of Washington, Seattle, WA, USA. ${ }^{7}$ University of Southern California, Los Angeles, CA, USA. ${ }^{8}$ Division of Pulmonary and Critical Care Medicine, University of Nevada School of Medicine, Las Vegas, NV, USA. 'Pulmonary and Critical Care Medicine, University of Alabama, Birmingham, AL, USA. ${ }^{10}$ Pulmonary, Allergy and Critical Care Medicine, Cleveland Clinic Foundation, Cleveland, OH, USA. ${ }^{11}$ Dept of Medicine, National Jewish Health, Denver, CO, USA. ${ }^{12}$ Dept of Pulmonary Disease, Erasmus MC, University Hospital Rotterdam, Rotterdam, The Netherlands. ${ }^{13}$ Dept of Pulmonary Medicine, Erasmus Medical Centre, University Hospital Rotterdam, Rotterdam, The Netherlands. ${ }^{14}$ Dept of Medicine, Texas Tech University Health Science Center, Lubbock, TX, USA. ${ }^{15}$ Biostatistics, Janssen Research and Development, LLC, Spring House, PA, USA. ${ }^{16} / \mathrm{mm}$ unology Biomarkers, Janssen Research and Development, LLC, Spring House, PA, USA. ${ }^{17}$ Immunology, Janssen Research and Development, LLC, Spring House, PA, USA.
\end{abstract}

Correspondence: Marc A. Judson, Division of Pulmonary and Critical Care Medicine, Dept of Medicine, MC-91, Albany Medical College, 47 New Scotland Avenue, Albany, New York 12208, NY, USA.

E-mail: judsonmamail.amc.edu

ABSTRACT Sarcoidosis is characterised by non-caseating granulomas that secrete pro-inflammatory cytokines, including interleukin (IL)-12, IL-23, and tumour necrosis factor (TNF)- $\alpha$. Ustekinumab and golimumab are monoclonal antibodies that specifically inhibit IL-12/IL-23 and TNF- $\alpha$, respectively.

Patients with chronic pulmonary sarcoidosis (lung group) and/or skin sarcoidosis (skin group) received either $180 \mathrm{mg}$ ustekinumab at week 0 followed by $90 \mathrm{mg}$ every 8 weeks, $200 \mathrm{mg}$ golimumab at week 0 followed by $100 \mathrm{mg}$ every 4 weeks, or placebo. Patients underwent corticosteroid tapering between weeks 16 and 28 . The primary end-point was week 16 change in percentage predicted forced vital capacity $(\Delta \mathrm{FVC} \%$ pred) in the lung group. Major secondary end-points were: week 28 for $\Delta \mathrm{FVC} \%$ pred, 6-min walking distance, St George's Respiratory Questionnaire (lung group), and Skin Physician Global Assessment response (skin group).

At week 16, no significant differences were observed in $\triangle \mathrm{FVC} \%$ pred with ustekinumab $(-0.15, \mathrm{p}=0.13)$ or golimumab (1.15, $\mathrm{p}=0.54)$ compared with placebo (2.02). At week 28, there were no significant improvements in the major secondary end-points, although a nonsignificant numerically greater Skin Physician Global Assessment response was observed following golimumab treatment (53\%) when compared with the placebo (30\%). Serious adverse events were similar in all treatment groups.

Although treatment was well tolerated, neither ustekinumab nor golimumab demonstrated efficacy in pulmonary sarcoidosis. However, trends towards improvement were observed with golimumab in some dermatological end-points.

@ERSpublications

Neither ustekinumab nor golimumab demonstrated efficacy for the treatment of patients with pulmonary sarcoidosis http://ow.ly/yaLt6 


\section{Introduction}

Sarcoidosis is a systemic, granulomatous disease with highly variable clinical manifestations, natural history, and prognosis that primarily affects the lungs and lymphatic systems [1]. Sarcoidosis is diagnosed by clinical findings and corroborating histological evidence of non-caseating epithelioid cell granulomas [2]. Over 90\% of patients have lung involvement [3], while skin lesions are observed in $\sim 25 \%$ of patients [4]. Although spontaneous remissions occur in almost two-thirds of patients, chronic or progressive disease is seen in $10-30 \%$ [5], with permanent pulmonary or extrapulmonary sequelae in at least $10-20 \%$ of patients. The disease is fatal in $1-5 \%$ of patients, typically due to progressive respiratory insufficiency, central nervous system, or myocardial involvement [5].

Currently, no therapy is approved for the treatment of sarcoidosis. The standard of care is oral corticosteroids (OCS), which has been shown to stabilise or improve the disease, although relapse commonly occurs once OCS therapy is tapered or discontinued [6]. Alternatives, such as antimalarial, cytotoxic, and biological agents, have shown variable efficacy [7], although treatment with these agents has also been associated with a relapse upon discontinuation, toxicity, and/or potentially serious adverse effects $[1,8]$. There is a substantial need for safer and more effective therapies for sarcoidosis.

Although the aetiology of sarcoidosis is unknown, the inflammatory cytokine, tumour necrosis factor (TNF) $-\alpha$, is instrumental to the formation and maintenance of the non-caseating granuloma that characterises sarcoidosis [9]. Macrophage inflammatory protein (MIP)-1 $\beta$, an immune cell chemoattractant (CCL4), is regulated by TNF- $\alpha$ [10] and higher levels of this inflammatory chemokine in some but not all sarcoidosis patients may indicate heightened activation of the TNF pathway in the subset of patients with the highest levels. TNF- $\alpha$ inhibition in patients with sarcoidosis has been shown to reduce disease signs and symptoms [11, 12]. In a phase II, multicentre, randomised, double-blind, placebo-controlled study of 138 patients with chronic sarcoidosis, treatment with infliximab (a chimeric monoclonal antibody that inhibits TNF- $\alpha$ ) resulted in a statistically significant improvement in the percentage predicted forced vital capacity (FVC) compared with placebo [1]. Improvement was also observed in other disease activity measures, such as chest radiograph reticulation (R) scores [13], MIP-1 $\beta$ levels [10], and serum angiotensin-converting enzyme (ACE) levels [1]. In a case series of 54 patients with lupus pernio, near or complete resolution was achieved in $77 \%$ of patients who received infliximab treatment compared with only $19 \%$ of patients receiving systemic corticosteroids [14].

Although the potential pathogenic role of excess TNF- $\alpha$ in granulomatous disease $[9,15-18]$ is well documented, TNF- $\alpha$ does not solely account for the T-helper cell type 1 (Th-1) dominated cytokine environment in the sarcoid lung; in particular, high levels of interferon (IFN)- $\gamma$ have also been implicated in the pathogenesis of sarcoidosis [19]. In addition, alveolar macrophages from patients with sarcoidosis have been shown to produce interleukin (IL)-12 in addition to TNF- $\alpha$, IL-1 $\beta$, and IL-6 [20-29]. Overexpression of IL-12, by activated alveolar macrophages, results in enhanced Th-1 cell proliferation and activation, leading to the increased expression of the pro-inflammatory mediators IFN- $\gamma$ and TNF- $\alpha$, and the secretion of cytokines and chemotactic factors that could, in turn, recruit and activate additional T-cells. Macrophages also produce profibrotic mediators, such as fibronectin [30], and transforming growth factor$\beta$ [31] that contribute to fibrotic granuloma development. Collectively, these data suggest that IL-12 may be involved in both initiating and perpetuating granulomatous inflammation of sarcoidosis. Recently, transcriptomic analysis of biopsies of sarcoid skin lesions demonstrated upregulation of TNF- $\alpha$ and IL-12 as well as IL-21 and IL-23, suggesting Th-1 and possibly Th-17 upregulation in sarcoidosis [32].Thus, inhibition of either TNF- $\alpha$ or IL-12/IL-23 could, potentially, be effective in the treatment of chronic sarcoidosis.

Golimumab is a human monoclonal antibody that binds TNF- $\alpha$ with high affinity and specificity and is approved for the treatment of moderate-to-severe rheumatoid arthritis, ulcerative colitis, active psoriatic

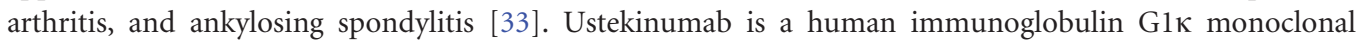
antibody that binds, with high affinity, to the shared p40 subunit of human IL-12 and IL-23 and is approved for the treatment of moderate-to-severe plaque psoriasis [34]. Here we report the results from a multicentre, randomised, double-blind, placebo-controlled study using ustekinumab or golimumab in patients with chronic pulmonary and/or cutaneous sarcoidosis.

Received: Jan 022014 | Accepted after revision: June 062014 | First published online: July 172014

This article has supplementary material available from erj.ersjournals.com

Clinical trial: This study is registered at www.clinicaltrials.gov with identifier number NCT00955279

Support statement: This study was supported by Janssen Research and Development, LLC, (Spring House, PA, USA).

Conflict of interest: Disclosures can be found alongside the online version of this article at erj.ersjournals.com 


\section{Material and methods \\ Patients}

Patients aged between 18 and 85 years were eligible for enrolment if they had histologically proven pulmonary or skin sarcoidosis diagnosed a minimum of 2 years before screening took place and no history of latent or active tuberculosis. In addition, a negative Quantiferon-GOLD assay was required at the screening visit in order for a patient to be enrolled. Entry into the lung group required evidence of lung parenchymal disease on a chest radiograph (stage II, III, or IV, but without cavitary disease), \% pred FVC 45-80\%, Medical Research Council dyspnoea score of $>2$, and a 6-min walking distance (6MWD) of between 100 and $550 \mathrm{~m}$. These criteria were chosen based on the results of a previous randomised, placebocontrolled trial of infliximab for chronic pulmonary sarcoidosis, where a significant response was observed and the subgroup of patients with the aforementioned criteria had a more robust response [1]. Entry into the skin group required: active, unresolved, chronic skin lesions for $\geqslant 3$ months, despite use of current systemic/local therapies; a single lesion of $\geqslant 2 \mathrm{~cm}$ or multiple lesions $(\geqslant 3)$ with at least one lesion $\geqslant 1 \mathrm{~cm}$ in size at its longest dimension; and a Skin Physician's Global Assessment (SPGA) score $\geqslant 2$. The SPGA score is based on the physician's assessment of induration and erythema of the patient's skin lesions, each graded on a 0 (none) to 4 (very severe) scale. Patients meeting entry criteria for both the lung and skin groups could be entered into both groups. All patients required treatment with $10-25 \mathrm{mg} \cdot \mathrm{day}^{-1}$ of prednisone or its equivalent orally and/or $\geqslant 1$ immunomodulator for $\geqslant 3$ months with a stable dose for $\geqslant 4$ weeks before screening. Key exclusion criteria were: a smoking history of $\geqslant 20$ pack-years; treatment with any other anti-TNF- $\alpha$ agent, anakinra, IL-12- or IL-23-targeted agent; previous cyclophosphamide use; local therapy or injections within 3 months or topical therapy within 1 month for skin sarcoidosis; clinically significant pulmonary hypertension receiving vasodilator therapy; and current or history of congestive heart failure.

This study was conducted according to the principles of the Declaration of Helsinki. The Institutional Review Board or Ethics Committee for each site approved the study and all patients provided written informed consent.

\section{Study design}

This was a phase II, multicentre, randomised, double-blind, placebo-controlled, three-arm study to evaluate the safety and efficacy of ustekinumab or golimumab in patients with chronic pulmonary and/or skin sarcoidosis. Following 1-4 weeks of screening, patients were randomly assigned in a 1:1:1 ratio stratified by baseline disease (pulmonary involvement only, skin involvement only, and both pulmonary and skin involvement) and prior anti-TNF- $\alpha$ biological use (yes/no) using an interactive voice-response system, to receive subcutaneous injections of ustekinumab, golimumab, or placebo. Patients assigned to ustekinumab received $180 \mathrm{mg}$ at week 0 and $90 \mathrm{mg}$ at weeks 8, 16, and 24 (with placebo at weeks 4, 12, and 20 to maintain blinding). Patients assigned to golimumab received $200 \mathrm{mg}$ at week 0 , followed by $100 \mathrm{mg}$ every 4 weeks through to week 24 (fig. 1). Patients receiving background OCS were maintained on a stable dose through week 16, if clinically possible, to enable the evaluation of the effect of treatment on the primary end-point on a stable background regimen. Then, if clinically stable, patients had their OCS dose tapered through week 28 , with dose reductions of $50 \%$ at weeks 16,20 , and 24 . Information on the dose of OCS and other sarcoid medications at baseline and post baseline are included in the online supplementary tables S1-S3.

The primary end-point was the change from baseline at week 16 in \% pred FVC in the lung group (i.e. those with lung only and lung and skin involvement). Major secondary end-points at week 28 were the change from baseline in the $6 \mathrm{MWD}(\Delta 6 \mathrm{MWD})$, the St. George's Respiratory Questionnaire (SGRQ) total score, percentage predicted FVC in the lung group, and the proportion of SPGA responders (score $\leqslant 1$, refer to online supplementary material) in the skin group (i.e. those with skin only and lung and skin involvement. The skin group was also assessed by the Sarcoidosis Activity and Severity Index (SASI), which was modified from BAUGHMAN et al. [35], (fig. S1). Samples were also collected at weeks 0, 4, 8, 16, 28, and 44 to explore the levels of ACE (R\&D Systems, Minneapolis, MN, USA), C-reactive protein (CRP) (Siemens Nephelometry, Deerfield, IL, USA), MIP-1 $\beta$ (MesoScale Discovery, Rockville, MD, USA), and other inflammatory biomarkers. An assessment of overall organ involvement, extrapulmonary physician organ severity tool (ePOST) [36], was performed at each visit, using all information available to the physician. Patients also completed several additional patient reported outcomes (PRO) questionnaires, including the 36-item short form health survey (SF-36) [37], the Fatigue Assessment Scale (FAS) [38], the sarcoidosis assessment tool (SAT) [39], and a patient global assessment (PGA) of overall wellbeing on a visual analogue scale. An Independent Data Monitoring Committee reviewed all available data after the 15th randomised patient had completed the week-16 visit and, periodically, thereafter. Patients were followed through to week 44 . 
a)
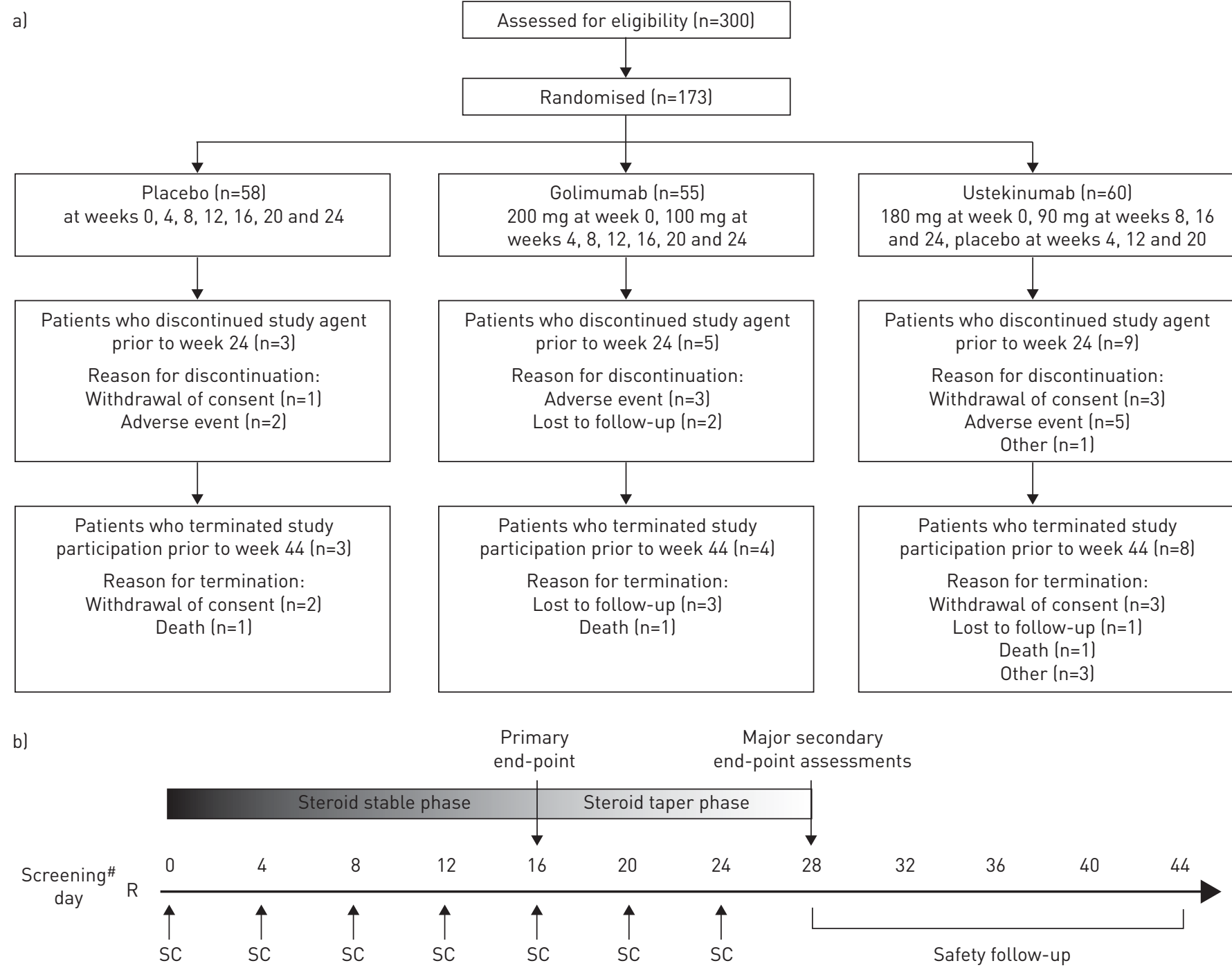

FIGURE 1 a) Study design and b) patient flow through the study. R: randomisation; SC: subcutaneous injections of study agent at weeks 0, 4, 8, 12, 16, 20 and 24. \#: screening from day -28 to day -1 .

\section{Statistical analyses}

The planned total sample size of 180 patients included a lung population of $\geqslant 135$ patients and a skin population of $\geqslant 45$ patients. Assuming a standard deviation of $7 \%$, 45 lung patients per treatment arm provided $86.4 \%$ power to detect a $5 \%$ difference in the change from baseline in \% pred FVC at week 16 among the three treatments at an overall 0.05 significance level, with Bonferroni adjustment. ANCOVA was used for the primary end-point analysis. The testing procedure comprised an overall test among placebo, ustekinumab, and golimumab, followed by two comparisons between ustekinumab and placebo, and golimumab and placebo. The significance level used for all analyses was 0.05 . The study would be considered positive if the overall test achieved statistical significance and at least one of the golimumab or ustekinumab arms was significantly better than placebo.

\section{Results}

\section{Baseline patient characteristics}

Between September 2009 and August 2012, 173 patients entered the study and were treated at 47 sites in nine countries. Of the 173 patients randomised, approximately half of the population $(50.9 \%, \mathrm{n}=88)$ was male and the majority were Caucasian $(61.3 \%, \mathrm{n}=106)$ (table 1$)$. A total of 15 patients terminated their study participation prior to the final study visit (placebo $n=3$, golimumab $n=4$, ustekinumab $n=8$ ) (fig. 1).

Baseline characteristics were well balanced across treatment arms. Approximately $67 \%$ of patients had lung involvement only, $24 \%$ had skin involvement only, and 10\% had both lung and skin involvement. 
TABLE 1 Baseline demographics and disease characteristics

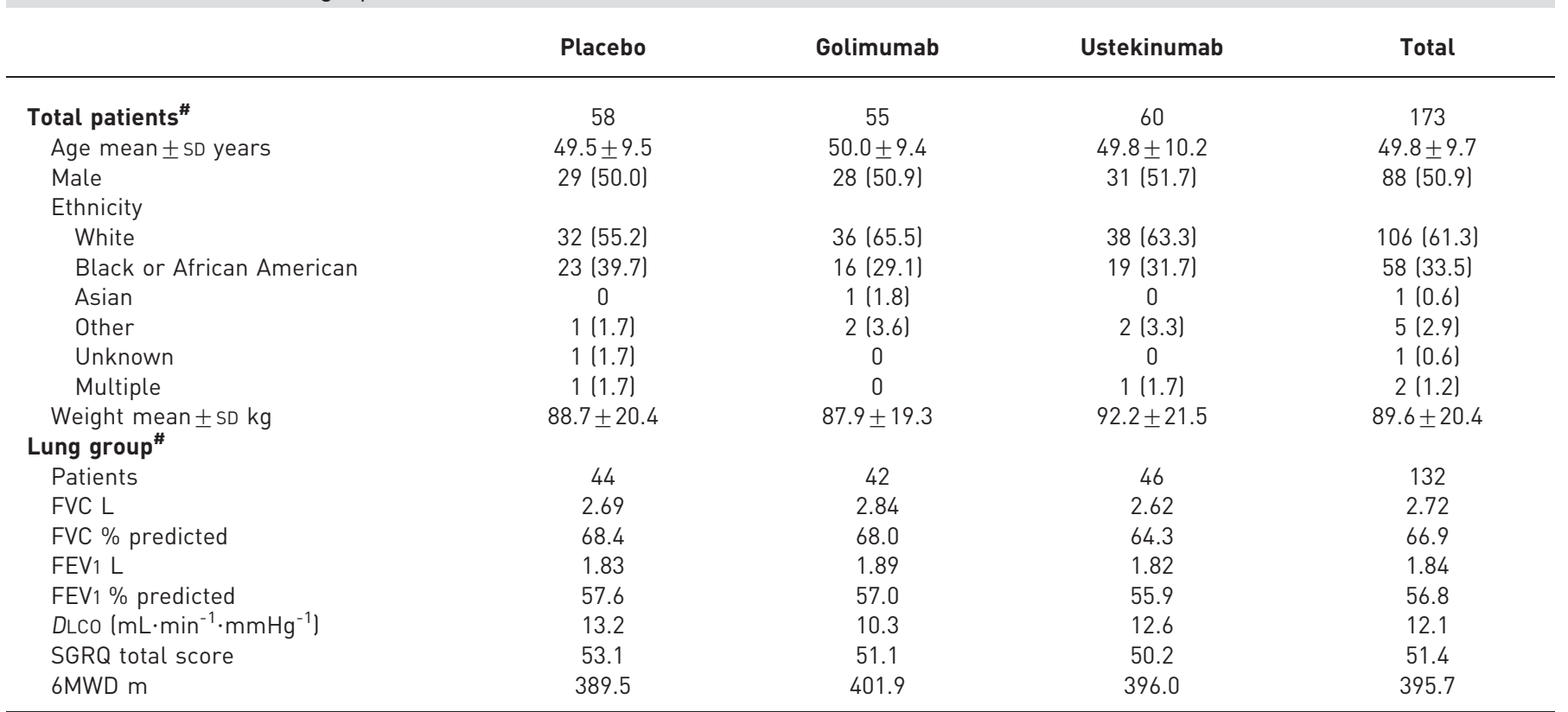

Data presented as $\mathrm{n}$ or $\mathrm{n}(\%)$, unless otherwise stated. FVC: forced vital capacity; FEV1: Forced expiratory volume in $1 \mathrm{~s}$; DLC0: diffusing capacity of the lung for carbon monoxide; SGRQ: St George's Respiratory Questionnaire; 6MWD: 6-min walking distance. \#: patients were randomised.

\section{Efficacy}

At week 16, there were no observed statistical differences in the primary end-point (the change from baseline in \% pred FVC) in the lung group with either active treatment compared to placebo (ustekinumab $-0.15, \mathrm{p}=0.126$; golimumab $1.15, \mathrm{p}=0.543$; placebo 2.02) (fig. 2 and table 2). The sensitivity and subgroup analyses on the primary end-point showed consistent results with the primary analysis (fig. S2) and included smoking status (yes/no), body mass index (BMI) ( $\geqslant 30$ versus $<30$ ), ethnicity (white/non-white), sex (male/female), and age ( $\geqslant 50$ versus $<50$ years). Of note is that patients with lower BMI tended to show improvement in FVC with golimumab treatment as opposed to patients with higher BMI, who tended toward worsening. An analysis of trough golimumab serum levels by quartiles, suggested that the change from baseline in FVC at week 16 tended to be increased in the two higher quartiles, but not the two lower quartiles (fig. S3). Subgroup analysis, based on the median levels of baseline biomarkers, failed to demonstrate any significant improvement in response in FVC (table S4).

At week 28, the active treatments demonstrated no statistically significant improvements compared with the placebo in any major secondary end-points (table 2). No significant differences were observed in the change

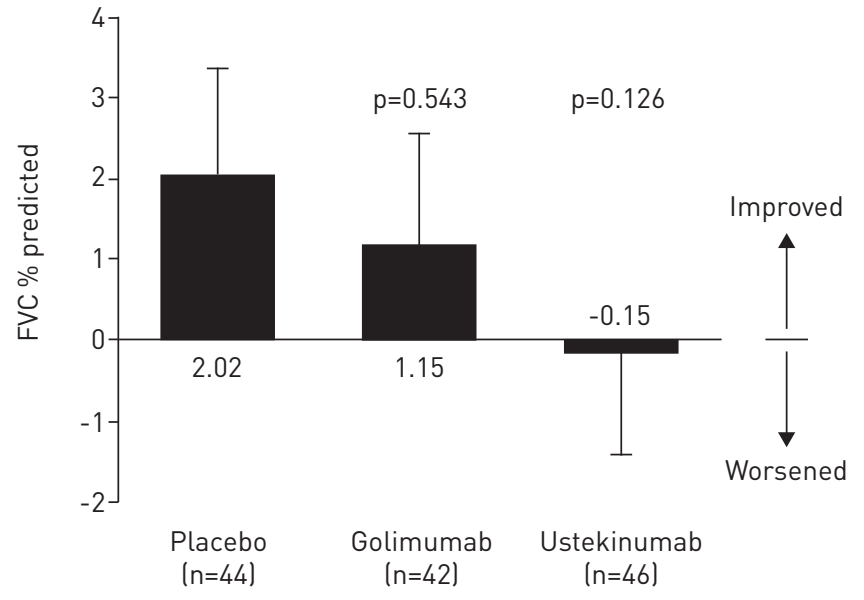

FIGURE 2 Primary end-point for the lung group at week 16 , the change from baseline in the mean $\pm \mathrm{SE} \%$ predicted forced vital capacity (FVC). Analysis uses last observation carried forward to input missing values. 


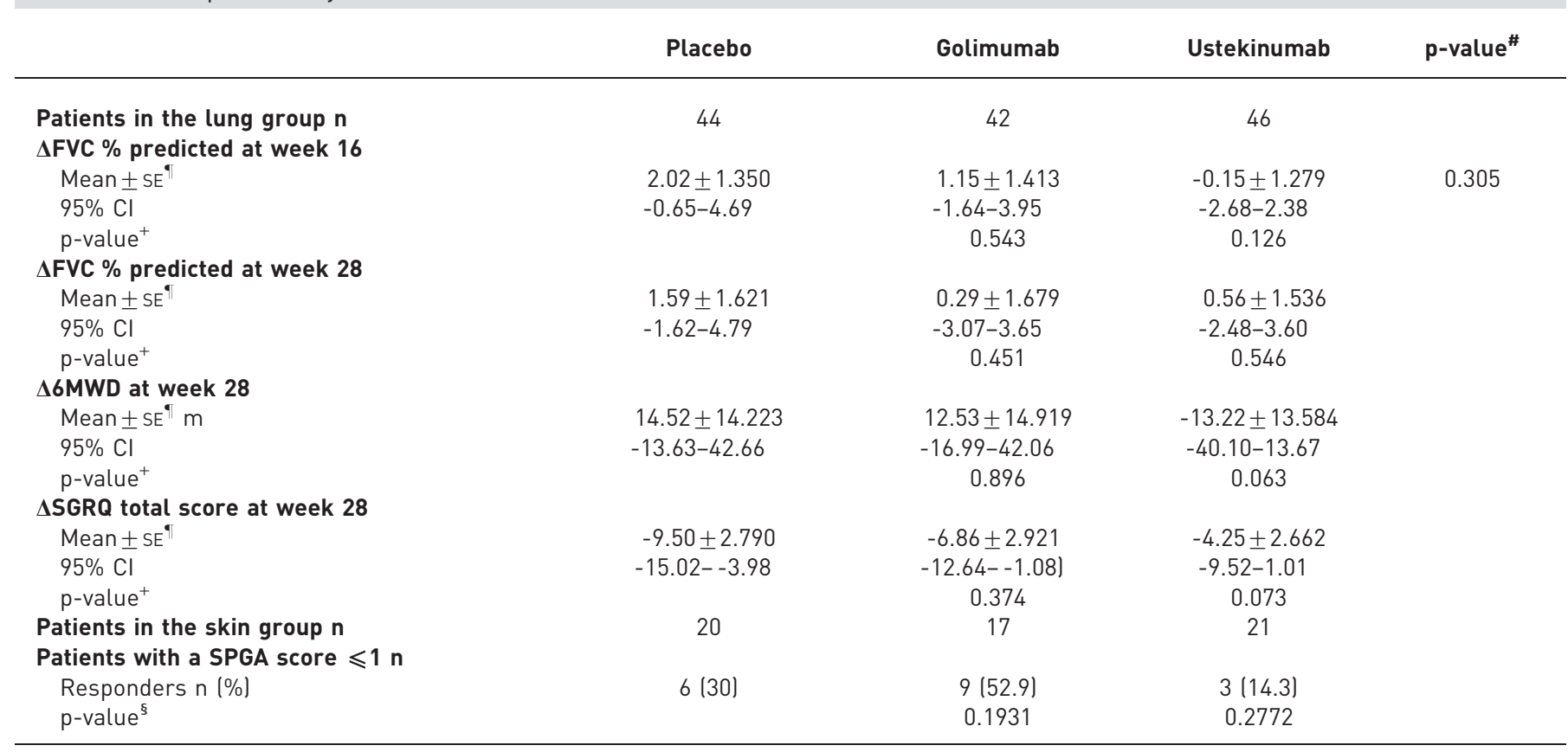

$\triangle \mathrm{FVC} \%$ : change from baseline in forced vital capacity; $\triangle 6 \mathrm{MWD}$ : change from baseline in 6-min walking distance; $\triangle \mathrm{SGRQ}$ : change from baseline in the St. George's Respiratory Questionnaire; SPGA: Skin Physician Global Assessment. \#: the overall p-value is based on the F-test; ${ }^{\top}$ : means are reported as least square means; ${ }^{+}$: treatment comparisons are based on linear contrasts; ${ }^{\S}$ : treatment comparisons are based on Fisher's exact test.

from baseline in \% pred FVC at week 28 in either of the active treatment arms (golimumab 0.29, $\mathrm{p}=0.451$; ustekinumab $0.56, \mathrm{p}=0.546$ ) compared to 1.59 for the placebo lung group. The $\triangle 6 \mathrm{MWD}$ was $12.53 \mathrm{~m}$ in the golimumab arm $(\mathrm{p}=0.896)$ and $-13.22 \mathrm{~m}$ in the ustekinumab arm $(\mathrm{p}=0.063)$ compared to $14.52 \mathrm{~m}$ in the placebo arm. At week 28, the mean change from baseline in the SGRQ total score in the lung group was: -9.50 for the placebo, -6.86 for golimumab $(p=0.374)$, and -4.25 for ustekinumab $(p=0.073)$. In addition, no significant improvements were observed in the active treatment arms relative to the placebo arm in the scores from the PRO questionnaires completed by the patients (SF-36, FAS, SAT, and PGA).

For the lung group who received OCS at baseline, a nominally greater proportion of golimumab-treated patients, but not ustekinumab-treated patients, were able to reduce their OCS dose by at least $50 \%$ during the taper-phase (golimumab 81.6\%, $\mathrm{p}=0.01$; ustekinumab $57.9 \%, \mathrm{p}=0.63$ ) compared with the placebo arm $(51.6 \%)$. For the golimumab arm, the results were mainly driven by patients with a BMI $<30$ (golimumab $85 \%$, placebo $36 \%, \mathrm{p}=0.0134$ ) consistent with the general trend towards improved results in patients with a lighter weight. Among the skin group, both arms tended to reduce OCS dose by at least 50\% (golimumab $70.0 \%, p=0.23$; ustekinumab $83.3 \%, p=0.047)$ compared with placebo $(40 \%)$. The proportion of patients in both the lung and skin groups with complete withdrawal of OCS use prior to week 28 was low and similar to that of the placebo: golimumab $11(28.9 \%)$ out of $38(\mathrm{p}=0.41)$, ustekinumab $7(18.4 \%)$ out of 38 $(\mathrm{p}=1.0)$, placebo $6(19.4 \%)$ out of 31 in the lung group; golimumab $3(30.0 \%)$ out of $10(\mathrm{p}=0.36)$, ustekinumab $1(8.3 \%)$ out of $12(\mathrm{p}=1.0)$, and placebo $2(13.3 \%)$ out of 15 in the skin group.

In the skin group, a nonsignificant improvement in SPGA response following golimumab treatment was observed $(52.9 \%, \mathrm{p}=0.19)$ compared with placebo $(30.0 \%)$, but not with ustekinumab treatment $(14.3 \%$, $\mathrm{p}=0.28$ ) (fig. 3a) at week 28. The target lesion score tended to improve with golimumab but not ustekinumab over time (golimumab $-2.3, \mathrm{p}=0.07$, ustekinumab $-1.2, \mathrm{p}=\mathrm{NS}$, placebo -1.4 ) at week 28 . A similar pattern was seen with SASI scores (golimumab -2.57, $\mathrm{p}=0.38$; ustekinumab $-0.50, \mathrm{p}=0.89$ ) compared with placebo (-0.52) (fig. 3b). Greater numerical improvement was also observed in the skin domain of the ePOST for golimumab-treated patients but not ustekinumab-treated patients (fig. 3c) $(\mathrm{p}=\mathrm{NS}$, for both $)$. In the skin group, there was a significant improvement in extrapulmonary involvement overall measured by the ePOST with golimumab, at weeks 20,24 , and 28 ( $\mathrm{p}=0.004,0.016,0.005$, respectively), but not with ustekinumab (fig. 3d). 

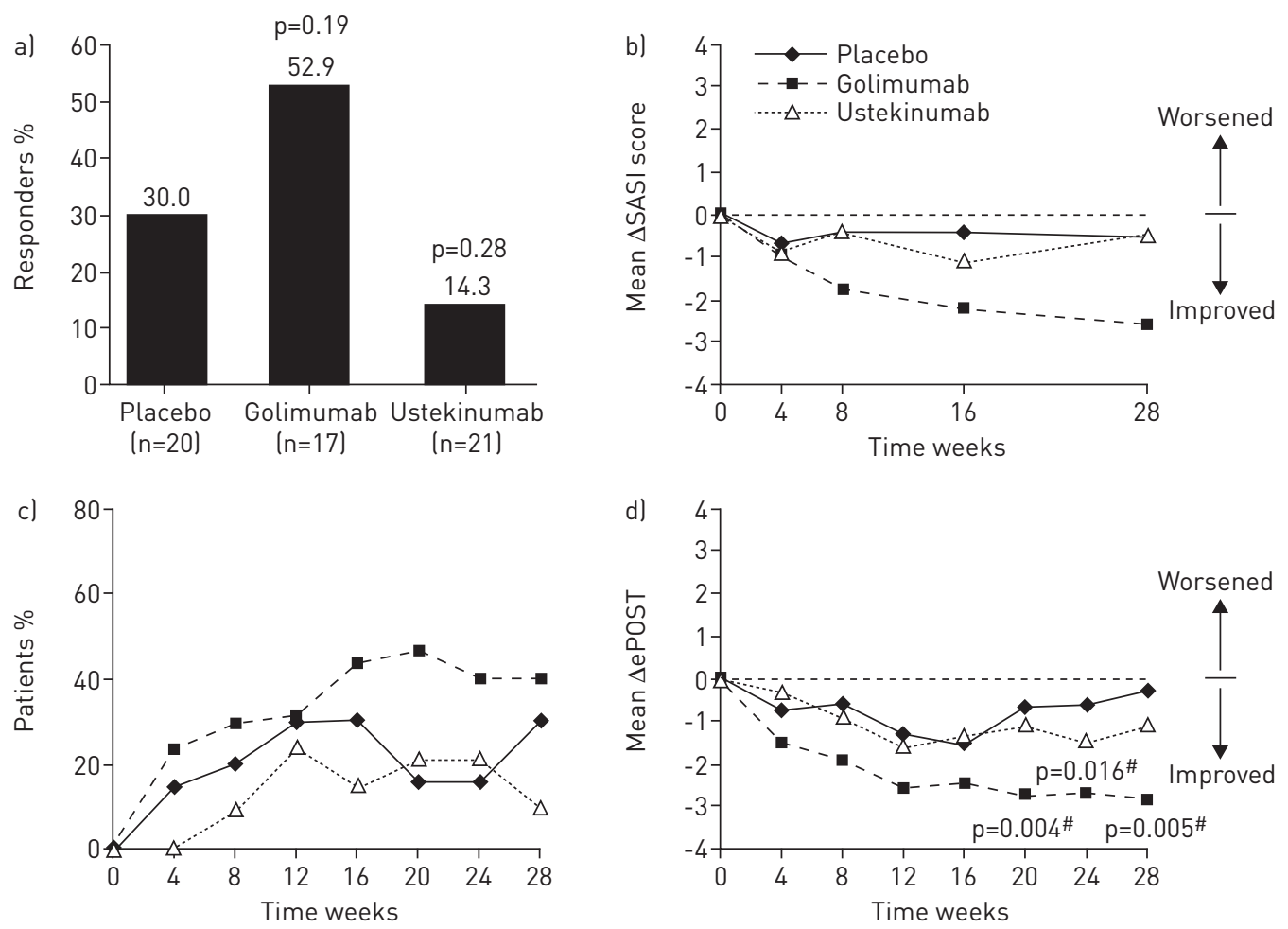

FIGURE 3 Skin group population assessments. a) Skin Physician Global Assessment Responders at week 28. b) Change from baseline in the Sarcoidosis Activity and Severity Index ( $\Delta$ SASI) score over time. c) Skin domain of the extrapulmonary Physician Organ Severity Tool (ePOST) defined as the percentage of patients with a score improvement of $\geqslant 2$ points over time. $\mathrm{d})$ Change from baseline in ePOST $(\Delta \mathrm{ePOST})$ over time. ${ }^{*}$ : nominal p-value versus placebo.

Of the serum inflammatory markers measured, (ACE, MIP-1 $\beta$, and CRP), MIP-1 $\beta$, an immune cell chemoattractant and pharmacodynamic marker of anti-TNF- $\alpha$ treatment, demonstrated a significant decrease from baseline by week 4 , and this difference was maintained through to week 28 , following treatment with golimumab (-84.57) when compared with placebo $(24.48,(\mathrm{p}<0.001)$. There was no difference observed with the treatment of ustekinumab (fig. 4). Statistically significant decreases in ACE were observed in both treatment arms at weeks 4,8 , and 16. ACE levels tended to rise in all 3 arms after week 16 during the steroid taper phase measured at week 28 (fig. S4).

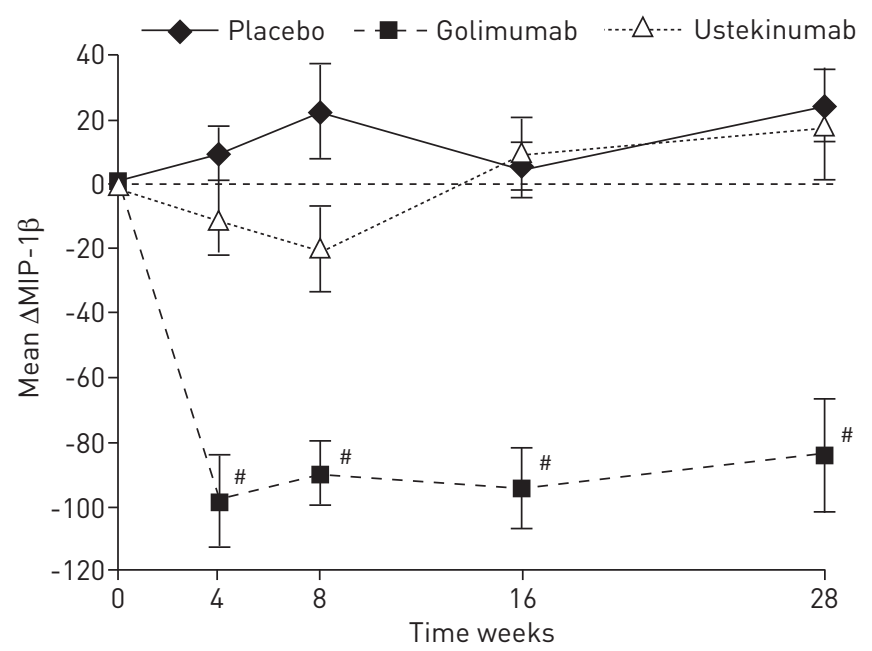

FIGURE 4 Macrophage inflammatory protein (MIP)-1 $\beta$ levels, response to treatment over time. ${ }^{\#}: \mathrm{p}<0.0001$ for golimumab versus placebo. $\Delta$ : change from baseline. 
Safety

Serious adverse events were reported throughout the course of the study in $15.5 \%(n=9), 12.7 \%(n=7)$, and $16.7 \%(n=10)$ for the placebo-treated, golimumab-treated, and ustekinumab-treated patients, respectively (table 3). The highest reported event was pneumonia (golimumab $n=1$, ustekinumab $n=3$ ). The occurrence of treatment emergent adverse events in patients throughout the course of the study were $93.1 \%$ $(n=54), 96.4 \%(n=53)$, and $93.3 \%(n=56)$ in the placebo-treated, golimumab-treated, and the ustekinumab-treated patients, respectively (table 4). Injection site reactions occurred in $3.4 \%(\mathrm{n}=2)$, $20 \%(n=11)$, and $5.0 \%(n=3)$ for placebo-treated, golimumab-treated, and ustekinumab-treated patients, respectively, and were mostly mild. There was no increased incidence of infection with either of the activetreatment arms of the study (golimumab 61.8\%, ustekinumab 65.0\%) compared with placebo arm (65.5\%). One case of tuberculosis was reported in a golimumab-treated patient.

Three deaths were reported during the study: a golimumab-treated patient died from sepsis 5 months after the first and only dose of golimumab, subsequent to being withdrawn from study drug administration due to dyspnoea; a ustekinumab-treated patient died due to acute respiratory failure 5 months after the last dose of ustekinumab at week 12; and one placebo-treated patient died, suddenly, 3 months after the last dose of study agent. Two reported malignancies included one event of recurrent squamous cell carcinoma (SCC) in a patient with a history of basal cell carcinoma and SCC (ustekinumab arm), and one case of breast cancer in a patient with a family history of breast cancer (placebo arm). One additional malignancy (chronic myeloid leukaemia) was reported in a female patient with a family history of malignancy, 2 months after completion of drug dosing (ustekinumab arm). Retrospective review of laboratory values and analyses of banked blood samples indicated that the malignancy was pre-existing at study entry and, therefore, was not recorded as a treatment-emergent event. There were no reports of possible anaphylactic reactions or possible delayed hypersensitivity (serum sickness-like) reactions through to week 44 .

\section{Discussion}

In this randomised, double-blind, placebo-controlled trial, neither golimumab nor ustekinumab demonstrated a significant effect on the primary end-point, the change from baseline at week 16 in $\%$ pred FVC at week 16 in the lung group, compared with the placebo. In addition, neither drug met any secondary study end-points at week $28(\triangle 6 \mathrm{MWD}, \triangle \mathrm{SGRQ}$ total score, $\triangle \mathrm{FVC} \%$ pred in the lung group, and the proportion of SPGA responders in the skin group). Additional sensitivity and subgroup analyses failed to demonstrate a significant difference between active study drug and placebo, although trends of improvement were consistently observed in the skin group end-points (SPGA, SASI, and ePOST) of patients treated with golimumab. Both golimumab and ustekinumab demonstrated a safety profile comparable to placebo in this cohort of patients with chronic sarcoidosis.

TABLE 3 Number of patients with $\geqslant 1$ treatment-emergent serious adverse events throughout the course of the study

Treated patients

Patients with events

Infections and infestations

Respiratory, thoracic and mediastinal disorders

Cardiac disorders

General disorders and administration site conditions

Metabolism and nutrition disorders

Musculoskeletal and connective tissue disorders

Psychiatric disorders

Gastrointestinal disorders

Immune system disorders

Injury, poisoning and procedural complications

Investigations

Neoplasms benign, malignant and unspecified, inclusive of cysts and polyps

Nervous system disorders

Renal and urinary disorders

Vascular disorders
Placebo

Golimumab

9 (15.5)

$3(5.2)$

1 (1.7)

0

$1(1.7)$

0

0

1 (1.7)

$1(1.7)$

1 (1.7)

1 (1.7)

0

1 (1.7)

$1(1.7)$

1 (1.7)

0

55
$7(12.7)$
$3(5.5)$
$3(5.5)$
$1(1.8)$
$1(1.8)$
$11(1.8)$
$11(1.8)$
$1(1.8)$
0
0
0
0
0
0
0
0

60

10 (16.7)

5 (8.3)

$1(1.7)$

$1(1.7)$

0

0

2 (3.3)

1 (1.7)

$1(1.7)$

1 (1.7)

0

$1(1.7)$

0

0

0

$1(1.7)$

Data presented as $\mathrm{n}$ or $\mathrm{n}(\%)$. Percentages were calculated with the number of patients treated in each arm as the denominator. Incidence is based on the number of patients experiencing at least one serious adverse event, not the number of events. 
TABLE 4 Number of patients with $\geqslant 1$ treatment-emergent adverse events $\geqslant 10 \%$ through the end of the study

\begin{tabular}{|c|c|c|c|}
\hline & Placebo & Golimumab & Ustekinumab \\
\hline Treated patients & 58 & 55 & 60 \\
\hline Patients with events & $54(93.1)$ & $53(96.4)$ & 56 (93.3) \\
\hline Cough & $15(25.9)$ & $11(20.0)$ & $13(21.7)$ \\
\hline Arthralgia & $16(27.6)$ & $13(23.6)$ & $9(15.0)$ \\
\hline Fatigue & 8 (13.8) & $11(20.0)$ & $12(20.0)$ \\
\hline Headache & $12(20.7)$ & $7(12.7)$ & $12(20.0)$ \\
\hline Upper respiratory tract infection & $13(22.4)$ & $6(10.9)$ & $10(16.7)$ \\
\hline Dyspnoea & $11(19.0)$ & $11(20.0)$ & $4(6.7)$ \\
\hline Diarrhoea & $11(19.0)$ & 6 (10.9) & $9(15.0)$ \\
\hline Bronchitis & 9 (15.5) & $8(14.5)$ & $8(13.3)$ \\
\hline Pain in extremity & $11(19.0)$ & $3(5.5)$ & $8(13.3)$ \\
\hline Back pain & 6 (10.3) & $6(10.9)$ & $9(15.0)$ \\
\hline Nausea & $6(10.3)$ & $7(12.7)$ & $8(13.3)$ \\
\hline Rash & $6(10.3)$ & $6(10.9)$ & $6(10.0)$ \\
\hline Sarcoidosis & $3(5.2)$ & $4(7.3)$ & $11(18.3)$ \\
\hline Sinusitis & 9 (15.5) & $3(5.5)$ & 5 (8.3) \\
\hline Oedema peripheral & $5(8.6)$ & $8(14.5)$ & $4(6.7)$ \\
\hline Vomiting & $7(12.1)$ & $4(7.3)$ & $6(10.0)$ \\
\hline Nasopharyngitis & $2(3.4)$ & $4(7.3)$ & $9(15.0)$ \\
\hline Skin lesion & 8 (13.8) & $1(1.8)$ & $6(10.0)$ \\
\hline Oropharyngeal pain & $4(6.9)$ & $2(3.6)$ & $7(11.7)$ \\
\hline
\end{tabular}

Data presented as $\mathrm{n}$ or $\mathrm{n}(\%)$. Percentages were calculated with the number of patients treated in each arm as the denominator. Incidence was based on the number of patients experiencing at least one adverse event, not the number of events.

The lack of efficacy of these two active drugs may provide insight into the immunopathogenesis and treatment of sarcoidosis. It is somewhat surprising that golimumab did not demonstrate a benefit versus placebo in this trial, as other TNF- $\alpha$ antagonists have shown favourable results for the treatment of sarcoidosis, including in randomised, double-blind, placebo-controlled trials [1, 14, 36, 40]. Furthermore, the entry criteria for the lung group in this trial matched that of a subgroup in a previous positive trial involving infliximab for pulmonary sarcoidosis that had a more robust response [1]. There are several potential explanations for the lack of response to golimumab in this trial. First, the limited clinical data available suggests that not all TNF- $\alpha$ antagonists are equally effective for sarcoidosis. In particular, etanercept has not been found to be particularly effective for various forms of sarcoidosis $[41,42]$ and has not been recommended for this condition [43]. Although limited data are available concerning the benefit of adalimumab for the treatment of sarcoidosis, it appears to require high dosing (administered subcutaneously) and to be administered over a longer period of time to maximize the benefit in comparison to infliximab, which is administered intravenously [43]. Secondly, it is possible that the dose of golimumab used in this trial was inadequate for the treatment of sarcoidosis. Although the additional sensitivity analyses were underpowered and conjectural, the fact that the cohort with a BMI $<30$ had a statistically nonsignificant greater improvement in FVC compared to those with a BMI $\geqslant 30$ suggests that the golimumab dose used may have been too low. This hypothesis is further supported by the tendency towards FVC improvement at higher golimumab trough levels (fig. S2). We also found that patients receiving golimumab with a lower BMI had a higher rate of corticosteroid tapering than the placebo arm. Thirdly, only a subset of sarcoidosis patients may have TNF- $\alpha$ driven disease. A subset analysis of the prior infliximab trial in pulmonary sarcoidosis showed better improvement in FVC in patients with measurable serum TNF- $\alpha$ levels at baseline (>lower limit of detection of $4 \mathrm{pg} \cdot \mathrm{mL}^{-1}$ ) [10]. The median serum TNF- $\alpha$ level in patients at baseline in the current study was $\sim 5 \mathrm{pg} \cdot \mathrm{mL}^{-1}$, and those with higher levels at baseline had slightly greater improvement in FVC, but this was not consistent across all inflammatory biomarkers (table S1). Fourthly, because corticosteroids also antagonise the action of TNF- $\alpha$, it is possible that the maintenance corticosteroid doses of the patients "masked" a benefit of golimumab. In support of this postulation, a recent re-analysis [44] of the positive infliximab trial for pulmonary sarcoidosis [1] demonstrated that patients receiving $\geqslant 15 \mathrm{mg} \cdot$ day $^{-1}$ of prednisone did not demonstrate an additional benefit from infliximab. This postulation may also explain why a nominally greater proportion of golimumab treated patients, in both the lung and skin groups, were able to reduce their corticosteroid dose by at least 50\% during the taper-phase compared with the placebo arm. Fifthly, the 3-month corticosteroid taper phase of the trial may have been too short to demonstrate a maximum corticosteroid-sparing effect, 
although the mean corticosteroid dose between weeks 28 and 44 (period whilst patient was not on study drug treatment) tended to be lower in both the golimumab $(7.2 \mathrm{mg}$ ) and ustekinumab (7.9 $\mathrm{mg})$ arms compared to the placebo arm $(12.2 \mathrm{mg})$. Finally, the skin group may have been inadequately powered to reach a conclusion concerning drug efficacy in this trial.

The lack of efficacy of ustekinumab was consistent, with no obvious signal of efficacy demonstrated in the primary end-point, secondary end-points, or sensitivity and subgroup analyses. This lack of efficacy may possibly indicate that the IL-12 p40 mechanism is not a major determinant of the disease process, although it is unknown if higher doses would have impacted on the disease. The lack of efficacy of ustekinumab must be reconciled against the findings that active sarcoidosis skin lesions showed upregulation of IL-12 and IL23 relative to control tissue [32] and increased IL-12 has been detected in sarcoidosis serum [26], bronchoalveolar lavage [25] and lymph nodes [45]. IL-12, in particular, has been thought to be a key driver of the dysregulation in interferon demonstrated in sarcoidosis [25, 32, 46]. Possibly, these mediators are not integral in driving interferon production or mediating sarcoid granuloma formation. Since ustekinumab blocks both IL-12 and IL-23, it leaves open the questions as to whether the blockade of IL-12 or IL-23 alone might be effective.

The design of this trial reflects a problem inherent in all novel drug therapy trials for sarcoidosis. Corticosteroids are highly effective for sarcoidosis. Because of the effectiveness of corticosteroids, it may be problematic to demonstrate an additional benefit of a study drug over the corticosteroid effect [44]. Because the enrolment criteria for this trial were identical to a subgroup that had a robust improvement in FVC in the previous infliximab trial, we believed that it was likely to see a benefit of at least golimumab in the current trial. We added a corticosteroid taper phase in the current trial in an attempt to uncover benefits of the study drugs that might have been obscured by maintenance corticosteroid therapy. Other study designs could have included tapering corticosteroids off or to a dose where the patient exacerbated before entering them in the trial. However, these approaches would have lengthened the duration of the trial. Possibly, biomarkers may be useful to determine drug responders as C-reactive protein identified responders to infliximab in the previous randomised, double-blind, corticosteroid-dependent pulmonary sarcoidosis trial [47].

Data from this study may give insight into the relative importance of immunopathogenic pathways in sarcoidosis. A number of samples collected during the course of the study are currently being analysed to evaluate the impact of treatment on the molecular signature of the disease and to identify alternative pathways with potential for therapeutic intervention. In addition, considering the wide spectrum in antisarcoidosis activity of the various TNF- $\alpha$ antagonists currently available, these data may lead to further modification of these agents to improve their efficacy or evaluation of the combination of anti-TNF- $\alpha$ with other key pathogenic mechanisms

In conclusion, although treatment with ustekinumab or golimumab was well tolerated, neither demonstrated efficacy in pulmonary sarcoidosis. However, trends towards improvement were observed with golimumab in some dermatological end-points. This trial may have been underpowered to fully examine the role of the study drugs in the skin group.

\section{Acknowledgements}

The authors and investigators greatly appreciate and acknowledge the patients who participated in this study. The authors also thank the Sarcoidosis Investigators and Robert Achenbach (Janssen Scientific Affairs, LLC, PA, USA ) for editorial and submission support of the manuscript.

The investigators for the study included: P. De Vuyst, C. Compere, W. Wuyts (Belgium); K.E. Bendstrup (Denmark); A. Bourdin, P. Chanez, B. Crestani, A. Tazi, D. Valeyre, B. Wallaert (France); U. Costabel, R. Bonnet, J. MüllerQuernheim, N. Schönfeld, U. Harnest (Germany); A. Millar, M. Whyte, S. Birring, T.M. Maher (UK); C. Albera, C. Saltini, A. Pesci, M. Luisetti, G. Paone, C. Agostini, P. Rottoli (Italy); R.E. Jonkers, M. Wijsenbeek, M. de Vries (The Netherlands); D. Ionita (Romania); R. Baughman, R. Burke, G. Davis, M. Judson, C. Strange, I. Huizar, M. Padilla, H. Shigemitsu, K. Gibson, N. Sweiss, E. White, D. Culver, M. Rosenbach, L. Maier, J. Barney, R. Enelow, E. Crouser, R. Gupta (USA).

\section{References}

1 Baughman RP, Drent M, Kavuru M, et al. Infliximab therapy in patients with chronic sarcoidosis and pulmonary involvement. Am J Respir Crit Care Med 2006; 174: 795-802.

Valeyre D, Prasse A, Nunes H, et al. Sarcoidosis. Lancet 2013; 383: 1155-1167.

Mihailovic-Vucinic V, Jovanovic D. Pulmonary sarcoidosis. Clin Chest Med 2008; 29: 459-473.

Sharma OP. Cutaneous sarcoidosis: clinical features and management. Chest 1972; 61: 320-325.

Statement on sarcoidosis. Am J Respir Crit Care Med 1999; 160: 736-755.

Gottlieb JE, Israel HL, Steiner RM, et al. Outcome in sarcoidosis: the relationship of relapse to corticosteroid therapy. Chest 1997; 111: 623-631.

7 Baughman RP, Nunes H, Sweiss NJ, et al. Established and experimental medical therapy of pulmonary sarcoidosis. Eur Respir J 2013; 41: 1424-1438. 
Vorselaars $\mathrm{AD}$, Verwoerd $\mathrm{A}$, van Moorsel $\mathrm{CH}$, et al. Prediction of relapse after discontinuation of infliximab therapy in severe sarcoidosis. Eur Respir J 2014; 43: 602-609.

9 Fehrenbach H, Zissel G, Goldmann T, et al. Alveolar macrophages are the main source for tumour necrosis factor- $\alpha$ in patients with sarcoidosis. Eur Respir J 2003; 21: 421-428.

10 Loza MJ, Brodmerkel C, Du Bois RM, et al. Inflammatory profile and response to anti-tumor necrosis factor therapy in patients with chronic pulmonary sarcoidosis. Clin Vaccine Immunol 2011; 18: 931-939.

11 Baughman RP, Costabel U, du Bois RM. Treatment of sarcoidosis. Clin Chest Med 2008; 29: 533-548.

12 Baughman RP, Iannuzzi M. Tumour necrosis factor in sarcoidosis and its potential for targeted therapy. BioDrugs 2003; 17: 425-431.

13 Baughman RP, Shipley R, Desai S, et al. Changes in chest roentgenogram of sarcoidosis patients during a clinical trial of infliximab therapy: comparison of different methods of evaluation. Chest 2009; 136: 526-535.

14 Stagaki E, Mountford WK, Lackland DT, et al. The treatment of lupus pernio: results of 116 treatment courses in 54 patients. Chest 2009; 135: 468-476.

15 Baughman RP, Strohofer SA, Buchsbaum J, et al. Release of tumor necrosis factor by alveolar macrophages of patients with sarcoidosis. J Lab Clin Med 1990; 115: 36-42.

16 Pueringer RJ, Schwartz DA, Dayton CS, et al. The relationship between alveolar macrophage TNF, IL-1, and PGE2 release, alveolitis, and disease severity in sarcoidosis. Chest 1993; 103: 832-838.

17 Steffen M, Petersen J, Oldigs M, et al. Increased secretion of tumor necrosis factor-alpha, interleukin-1-beta, and interleukin-6 by alveolar macrophages from patients with sarcoidosis. J Allergy Clin Immunol 1993; 91: 939-949.

18 Ziegenhagen MW, Benner UK, Zissel G, et al. Sarcoidosis: TNF-alpha release from alveolar macrophages and serum level of sIL-2R are prognostic markers. Am J Respir Crit Care Med 1997; 156: 1586-1592.

19 Robinson BW, McLemore TL, Crystal RG. Gamma interferon is spontaneously released by alveolar macrophages and lung T lymphocytes in patients with pulmonary sarcoidosis. J Clin Invest 1985; 75: 1488-1495.

20 Antoniou KM, Tzouvelekis A, Alexandrakis MG, et al. Upregulation of Th1 cytokine profile (IL-12, IL-18) in bronchoalveolar lavage fluid in patients with pulmonary sarcoidosis. J Interferon Cytokine Res 2006; 26: 400-405.

21 Barbarin V, Petrek M, Kolek V, et al. Characterization of p40 and IL-10 in the BALF of patients with pulmonary sarcoidosis. J Interferon Cytokine Res 2003; 23: 449-456.

22 Bergeron A, Bonay M, Kambouchner M, et al. Cytokine patterns in tuberculous and sarcoid granulomas: correlations with histopathologic features of the granulomatous response. J Immunol 1997; 159: 3034-3043.

23 Meloni F, Caporali R, Marone Bianco A, et al. BAL cytokine profile in different interstitial lung diseases: a focus on systemic sclerosis. Sarcoidosis Vasc Diffuse Lung Dis 2004; 21: 111-118.

24 Moller DR, Forman JD, Liu MC, et al. Enhanced expression of IL 12 associated with Th1 cytokine profiles in active pulmonary sarcoidosis. J Immunol 1996; 156: 4952-4960.

25 Shigehara K, Shijubo N, Ohmichi M, et al. IL-12 and IL-18 are increased and stimulate IFN- $\gamma$ production in sarcoid lungs. J Immunol 2001; 166: 642-649.

26 Shigehara K, Shijubo N, Ohmichi M, et al. Increased circulating interleukin-12 (IL-12) p40 in pulmonary sarcoidosis. Clin Exp Immunol 2003; 132: 152-157.

27 Shigehara K, Shijubo N, Ohmichi M, et al. Enhanced mRNA expression of Th1 cytokines and IL-12 in active pulmonary sarcoidosis. Sarcoidosis Vasc Diffuse Lung Dis 2000; 17: 151-157.

28 Minshall EM, Tsicopoulos A, Yasruel Z, et al. Cytokine mRNA gene expression in active and nonactive pulmonary sarcoidosis. Eur Respir J 1997; 10: 2034-2039.

29 Taha RA, Minshall EM, Olivenstein R, et al. Increased expression of IL-12 receptor mRNA in active pulmonary tuberculosis and sarcoidosis. Am J Respir Crit Care Med 1999; 160: 1119-1123.

30 Rennard SI, Hunninghake GW, Bitterman PB, et al. Production of fibronectin by the human alveolar macrophage: mechanism for the recruitment of fibroblasts to sites of tissue injury in interstitial lung diseases. Proc Natl Acad Sci USA 1981; 78: 7147-7151.

31 Roman J, Jeon YJ, Gal A, et al. Distribution of extracellular matrices, matrix receptors, and transforming growth factor- $\beta 1$ in human and experimental lung granulomatous inflammation. Am J Med Sci 1995; 309: 124-133.

32 Judson MA, Marchell RM, Mascelli M, et al. Molecular profiling and gene expression analysis in cutaneous sarcoidosis: the role of interleukin-12, interleukin-23, and the T-helper 17 pathway. J Am Acad Dermatol 2012; 66: 901-910.

33 US National Library of Medicine. Simponi (golimumab) solution (Jannssen Biotech, Inc.). http://dailymed.nlm. nih.gov/dailymed/lookup.cfm?setid=9e260a47-55af-4c92-8d88-a86ccc767fff Date last accessed: June 17, 2014. Date last updated: February, 2014

34 US National Library of Medicine. Stelara (ustekinumab) injection, solution (Janssen Biotech, Inc.) http://dailymed. nlm.nih.gov/dailymed/lookup.cfm?setid=c77a9664-e3bb-4023-b400-127aa53bca2b Date last accessed: June 17, 2014. Date last updated: March, 2014

35 Baughman RP, Judson MA, Teirstein A, et al. Chronic facial sarcoidosis including lupus pernio: clinical description and proposed scoring systems. Am J Clin Dermatol 2008; 9: 155-161.

36 Judson MA, Baughman RP, Costabel U, et al. Efficacy of infliximab in extrapulmonary sarcoidosis: results from a randomised trial. Eur Respir J 2008; 31: 1189-1196.

37 Ware JE Jr, Sherbourne CD. The MOS 36-item short-form health survey (SF-36). I. Conceptual framework and item selection. Med Care 1992; 30: 473-483.

38 De Vries J, Michielsen H, Van Heck GL, et al. Measuring fatigue in sarcoidosis: the Fatigue Assessment Scale (FAS). Br J Health Psychol 2004; 9: 279-291.

39 Victorson DE, Cella D, Grund H, et al. A conceptual model of health-related quality of life in sarcoidosis. Qual Life Res 2014; 23: 89-101.

40 Pariser RJ, Paul J, Hirano S, et al. A double-blind, randomized, placebo-controlled trial of adalimumab in the treatment of cutaneous sarcoidosis. J Am Acad Dermatol 2013; 68: 765-773.

41 Baughman RP, Lower EE, Bradley DA, et al. Etanercept for refractory ocular sarcoidosis: results of a double-blind randomized trial. Chest 2005; 128: 1062-1067.

42 Utz JP, Limper AH, Kalra S, et al. Etanercept for the treatment of stage II and III progressive pulmonary sarcoidosis. Chest 2003; 124: 177-185. 
43 Baughman RP, Lower EE, Drent M. Inhibitors of tumor necrosis factor (TNF) in sarcoidosis: who, what, and how to use them. Sarcoidosis Vasc Diffuse Lung Dis 2008; 25: 76-89.

44 Judson MA, Baughman RP, Costabel U, et al. The potential additional benefit of infliximab in patients with chronic pulmonary sarcoidosis already receiving corticosteroids: a retrospective analysis from a randomized clinical trial. Respir Med 2014; 108: 189-194.

45 Hata M, Sugisaki K, Miyazaki E, et al. Circulating IL-12 p40 is increased in the patients with sarcoidosis, correlation with clinical markers. Intern Med 2007; 46: 1387-1393.

46 Koth LL, Solberg OD, Peng JC, et al. Sarcoidosis blood transcriptome reflects lung inflammation and overlaps with tuberculosis. Am J Respir Crit Care Med 2011; 184: 1153-1163.

47 Sweiss NJ, Barnathan ES, Lo K, et al. C-reactive protein predicts response to infliximab in patients with chronic sarcoidosis. Sarcoidosis Vasc Diffuse Lung Dis 2010; 27: 49-56. 